\title{
Universal and Near-Universal Cycles of Set Partitions
}

\author{
Zach Higgins* \\ Department of Mathematics \\ University of Florida, U.S.A \\ zhiggins11@yahoo.com \\ Bertilla Sieben ${ }^{\ddagger}$ \\ Department of Mathematics \\ Princeton University, U.S.A. \\ bsieben@princeton. edu
}

\author{
Elizabeth Kelley ${ }^{\dagger}$ \\ Department of Mathematics \\ University of Minnesota, U.S.A. \\ ekelley@g.hmc.edu \\ Anant Godbole ${ }^{\S}$ \\ Department of Mathematics and Statistics \\ East Tennessee State University, U.S.A.
}

godbolea@etsu.edu

Submitted: Feb 20, 2015; Accepted: Dec 17, 2015; Published: Dec 23, 2015

Mathematics Subject Classifications: 05B30, 05A18

\begin{abstract}
We study universal cycles of the set $\mathcal{P}(n, k)$ of $k$-partitions of the set $[n]:=$ $\{1,2, \ldots, n\}$ and prove that the transition digraph associated with $\mathcal{P}(n, k)$ is Eulerian. But this does not imply that universal cycles (or ucycles) exist, since vertices represent equivalence classes of partitions. We use this result to prove, however, that ucycles of $\mathcal{P}(n, k)$ exist for all $n \geqslant 3$ when $k=2$. We reprove that they exist for odd $n$ when $k=n-1$ and that they do not exist for even $n$ when $k=n-1$. An infinite family of $(n, k)$ for which ucycles do not exist is shown to be those pairs for which $\left\{\begin{array}{l}n-2 \\ k-2\end{array}\right\}$ is odd $(3 \leqslant k<n-1)$. We also show that there exist universal cycles of partitions of $[n]$ into $k$ subsets of distinct sizes when $k$ is sufficiently smaller than $n$, and therefore that there exist universal packings of the partitions in $\mathcal{P}(n, k)$. An analogous result for coverings completes the investigation.
\end{abstract}

\section{Introduction}

A universal cycle, or ucycle, is a cyclic ordering of a set of objects $\mathcal{C}$, each represented as a string of length $N$. The ordering requires that object $b=b_{0} b_{1} \ldots b_{N-1}$ follow object

\footnotetext{
*Supported by NSF Grant 1263009

${ }^{\dagger}$ Supported by NSF Grant 1263009

${ }^{\ddagger}$ Supported by NSF Grant 1263009

${ }^{\S}$ Supported by NSF Grant 1263009
} 
$a=a_{0} a_{1} \ldots a_{N-1}$ only if $a_{1} a_{2} \ldots a_{N-1}=b_{0} b_{1} \ldots b_{N-2}$. These were originally introduced in 1992 by Chung, Diaconis, and Graham [2] as generalizations of de Bruijn cycles. As an example, the cyclic string 112233 encodes each of the six multisets of size 2 from the set $\{1,2,3\}$. Another well-quoted example, from [9], is the string

\section{8 ,}

where each block is obtained from the previous one by addition of 5 modulo 8 . This string is an encoding of the $56=\left(\begin{array}{l}8 \\ 3\end{array}\right) 3$-subsets of the set $[8]:=\{1,2,3,4,5,6,7,8\}$. A seminal paper in the area is that of Chung, Diaconis and Graham [2] who studied ucycles of

- subsets of size $k$ of an $n$-element set (as in the above example);

- set partitions (the focus of this paper); and

- permutations (with a necessarily augmented ground set and the use of order isomorphism representations, e.g., the string 124324 encodes each of the six permutations of $[3]=\{1,2,3\}$ in an order isomorphic fashion, which is clearly not possible to do using the ground set [3]).

In [2] it is shown that for $n \geqslant 4$, there does exist a ucycle of all partitions $\mathcal{P}(n)$ of the set $[n]$ into an arbitrary number of parts. For example, we have the ucycle abcbccccddcdeec of $\mathcal{P}(4)$, where, for example, the substring dcde encodes the partition $13|2| 4$. Note that the alphabet used was, in this case, of size 5 , though an alphabet of (minimum) size 5 is shown to suffice to encode $\mathcal{P}(5)$ as

\section{DDDDDCH H HCCDDCCCHCHCSH HSDSSDSSHSDDCH}

\section{SSCHSHDHSCHSJCDC.}

The above example reflects tongue-in-cheek humor, since there are 52 partitions of [5] and the above ucycle has 13 cards of each suit - except that one spade has been replaced by a joker! The authors of [2] also ask how many partitions of $\mathcal{P}(n)$ using an alphabet of size $N \geqslant n$ exist. This question will also be of deep relevance to us, as alluded to in the later section on Open Problems.

As noted in [6], however, not much seems to be known about ucycles of the partitions $\mathcal{P}(n, k)\left(|\mathcal{P}(n, k)|=\left\{\begin{array}{l}n \\ k\end{array}\right\}\right)$ of $[n]$ into $k$ parts. In this paper, a $k$-partition of $[n]$ is represented as a string $s_{1}, s_{2} \ldots s_{n}$ over the alphabet $[k]=\{1,2, \ldots, k\}$, where $i$ and $j$ are in the same block if and only if $s_{i}=s_{j}$. In general this representation is not unique and it is used in different ways in Sections 2 and 3. In [4], it was shown that for $k=n-1$, ucycles exist if and only if $n$ is odd. At the other end of the $k$-spectrum, the authors of [7] showed that for odd $n$, one could find a ucycle of partitions of $[n]$ into two parts, and that an "asymptotically good universal packing" could be found for $k=3$, i.e., that there was a string of length $T(n, 3)<\left\{\begin{array}{l}n \\ 3\end{array}\right\}$ with

$$
\frac{T(n, 3)}{\left\{\begin{array}{l}
n \\
3
\end{array}\right\}} \rightarrow 1, \quad n \rightarrow \infty,
$$


where each of the $T(n, 3)$ consecutive strings of length $n$ represented a different partition of $[n]$ into 3 parts of distinct sizes. The authors of [7] also proved that ucycles of partitions of $[n]$ into 2 parts exist if $n=4$ or 6 , as evidenced by the respective explicit examples

$a a b b a b a$

and

abaaaabbaababbbabbaaabbbbbababa.

It is this work that we build on. In Section 2, we generalize the above result on asymptotically good universal packings (upackings) to the case of all fixed $k$ as $n \rightarrow \infty$, as well as to ucoverings, which are also shown to be "asymptotically good." Finally, Section 3 is devoted to showing that the transition digraph associated with $\mathcal{P}(n, k)$ is Eulerian. As noted in the work of [2], this does not necessarily imply that universal cycles exist, since the digraph vertices represent equivalence classes of partitions. We use our result to prove, however, that ucycles of $\mathcal{P}(n, k)$ exist for all $n \geqslant 3$ when $k=2$ and for odd $n$ when $k=n-1$, the latter recovering the result in [4]. We also (re)prove that ucycles do not exist for $n$ even when $k=n-1$. Finally, we show that for even $n$, ucycles do not exist when $\left\{\begin{array}{l}n-2 \\ k-2\end{array}\right\}$ is odd $(3 \leqslant k<n-1)$. There are infinitely many such pairs of values $(n, k)$ for $k \not \equiv 1(\bmod 4)$. Moreover, the technique we exhibit in Section 3 has the potential to tease out many more results along these lines.

\section{Universal Packings and Coverings of Partitions of $[n]$ into $k$ parts}

One of the main results in [10] was that one could create a ucycle of all surjections from $[n]$ to $[k]$ iff $n>k$. Since there are $k$ ! surjections that yield the same set partition, we need to be more careful, and proceed by showing in Theorem 1 that for sufficiently large $n$, it is possible to ucycle partitions of $[n]$ into $k$ parts of distinct sizes. We represent such partitions as surjections from $f:[n] \rightarrow[k] ; n>k$, with $1 \leqslant\left|f^{-1}(\{1\})\right|<\left|f^{-1}(\{2\})\right|<$ $\ldots<\left|f^{-1}(\{k\})\right|$. The fact that asymptotically good upackings exist is proved in Corollary 4.

Theorem 1. For each fixed $k, n ; n \geqslant \frac{(k+4)(k-1)}{2}+1$, there exists a ucycle of all onto functions $f:[n] \rightarrow[k]$ such that the preimage cardinality function $\left|f^{-1}\right|:\{\{1\},\{2\}, \ldots\{k\}\} \rightarrow$ $[n]$ is strictly increasing.

Proof. Following the usual process used to exhibit existence of deBruijn cycles (see, e.g., [6]), we create a digraph $D$ in which the vertices are sequences of length $n-1$, of numbers in $\{1, \ldots, k\}$, for which there exists at least one number in $\{1, \ldots, k\}$ which, when added as a prefix or a suffix to the vertex label creates a sequence of length $n$ which, using the special canonical format we have adopted, represents a partition of $[n]$ into $k$ parts of distinct sizes. For example, with $n=10, k=4,122333444$ is a legal vertex, as is 123334444. On the other hand, 112233344 is not in the underlying graph. There is a directed edge from a vertex $v=v_{1}, \ldots, v_{n-1}$ to another vertex $w=w_{1} \ldots w_{n-1}$ if 
$v_{i}=w_{i-1}$ for each $i=2,3, \ldots, n-1$ and $v_{1}, \ldots, v_{n-1} w_{n-1}$ represents a partition of $[n]$ into $k$ distinct parts. The edges of the digraph, labeled by vertex label concatenation, are thus sequences representing partitions of $[n]$ into $k$ distinct parts. It is important to mention here that the definition of edges is different in Section 3. Here edge labels must have, for each $1 \leqslant i \leqslant k-1$, more of the letters $i+1$ than the letters $i$, whereas in Section 3 any of the $k$ ! representations may be used to label a set partition.

The problem of finding a ucycle is reduced to the problem of finding an Eulerian circuit in this digraph. We know that Eulerian circuits exist if the graph is both weakly connected and balanced, i.e., for each vertex $v$, the in- and out-degrees of $v$ are equal.

If a number from 1 through $k$ can be added as a prefix to a vertex label, then it can also be added to the end as a sequence suffix, since it is only the numbers of 1 's, 2's, ... and $k$ 's that actually matter in determining if an edge represents a partition into distinct parts. Therefore, the digraph is balanced, though we note that the in- and out-degrees, while equal, are quite different at different vertices. We call the common value that guarantees balancedness the degree. For example for $k=3$, the vertex 123333333 has degree 1; the vertex 122333333 has degree 2; and the vertex 122233333 has degree 3. In general, one may write down a formula for $\operatorname{deg}(v)$ depending on the differences between the number of $i+1 \mathrm{~s}$ and the number of $i$ s in $v$.

To show that the digraph is weakly connected, we will show that it is possible to reach the designated target vertex $22333 \ldots(k-1) \ldots(k-1) k \ldots k$ (with $j j$ 's for each $2 \leqslant j \leqslant k-1$, and $n-\frac{k(k-1)}{2} k$ 's) from any other starting vertex. In fact, we will show something stronger, namely that one can traverse from any edge to the edge $122333 \ldots(k-$ $1) \ldots(k-1) k \ldots k$ from whence we may reach the target vertex in a single step. Notice that edges represent legal partitions in $\mathcal{P}(n, k)$ only if $n \geqslant n_{0}:=k(k+1) / 2$, and that this is satisfied for the target vertex. In what follows, when we say that we "switch" a number $j$ in a given position to another number $j^{\prime}$, we mean that after possibly rotating the word that represents the edge so as to maintain cyclic order, we replace an edge that begins with $j$ to one that ends in $j^{\prime}$.

Claim 2. We are able to "switch" any $j \in\{2,3, \ldots, k-1\}$ into a $k$ and vice versa (possibly through several steps) provided that $n \geqslant n_{1}:=\frac{(k+4)(k-1)}{2}+1=n_{0}+(k-1)$, where the "extra" $(k-1)$ digits allow us to make the switches as needed without violating monotonicity.

Proof. Let a partition size vector (PSV) be a numerical partition of the integer $n$ into $k$ distinct parts. Each PSV can be used to express the number of 1's, 2's, etc., in order, of an edge. If $n$ is too low, the values of the PSVs might not allow the above-mentioned switch from an integer $j$ to or from $k$ to happen; for example, if $n=18 ; k=5$, then the only possible PSV's are $(1,2,3,4,8) ;(1,2,3,5,7)$; and $(1,2,4,5,6)$. A 2 cannot be changed to a 5 directly. If we attempt to increase the number of 2's to three (by decreasing the number of 5's) to facilitate this change, this too causes problems with monotonicity. However, if we have $n=19$ and $k=5$, the PSV's are $(1,2,3,4,9),(1,2,3,5,8),(1,2,3,6,7),(1,2,4,5,7)$, and $(1,3,4,5,6)$, which will be seen to imply that no matter what our starting position, 
we have enough "spaces" so that we can switch a 2, 3, or a 4 into a 5 (possibly in multiple steps) and back again eventually. Notice that for $k=5, n_{1}=19$.

Given an edge of weight $n_{1}:=\frac{(k+4)(k-1)}{2}+1$, the sums of the gaps between the components of the PSV may be as low as $k$, corresponding to the PSV $(1,3,4, \ldots, k+1)$, or as high as $2 k-2$, corresponding to the $\operatorname{PSV}(1,2, \ldots, k-1,2 k-1)$. To change a $j$ into a $k$ we cannot have the number of $j$ s be one more than the number of $j-1$ s or one less than the number of $j+1 \mathrm{~s}$. Notice first that, by the above discussion, there must be a gap of two somewhere in the PSV, which we represent by $\left(\rho_{1}, \rho_{2}, \ldots, \rho_{k}\right)$. Suppose first that $i$ is the largest integer for which $\rho_{i}+2 \leqslant \rho_{i+1}$ and that $j \geqslant i+1$, where $j$ is the integer that we wish to change into a $k$. Then we move the gap sequentially closer to $j$ by successively replacing integers between $i+1$ and $j-1$ by $k$. This creates a gap of two between $\rho_{j-1}$ and $\rho_{j}$, allowing us to switch a $j$ with a $k$ as desired. The process is a little different if $j \leqslant i$, where the leftmost gap of two in the PSV is between $\rho_{i}$ and $\rho_{i+1}$; in this case we first change the PSV to $(1,2, \ldots, k-1, \ell)$ where $\ell \geqslant 2 k-1$, and then to $\left(1,2, \ldots, j-1, j+1, j+2, \ldots, k, \ell^{\prime}\right)$, where $\ell^{\prime} \geqslant k+1$. We can now replace $j$ by $k$. Replacing a $k$ by a $j$ follows a similar and inverted process. This proves the claim.

To continue with the proof of Theorem 1, our algorithm to reach the target sequence will be to first, if we have more than one 1, change all extra 1's into $k$ 's. We will then "underline" the single remaining 1 as something we won't touch again. Next, we will switch the number to the right of the 1 into a $k$, possibly in multiple steps, and then the $k$ back into a 2, again possibly through multiple steps. We will now underline the 1 and the 2 together, as something we won't touch again. Next we will consider the next number to the right of this 2 and switch it to a $k$, and then switch back from the $k$ into a 2 again, then underline the sequence 122, and switch all remaining 2's in the sequence into $k$ 's. Next, we consider the number to right of the second 2 , switch it into a $k$, and then switch from the $k$ back into a 3 , then underline 1223, etc., until we reach the target sequence.

The next theorem shows that there exists a ucovering of all partitions of $[n]$ into $k$ parts if $n$ is large enough; for simplicity we let the threshold $n$ be the same as in Theorem 1 . This is because any partition may be represented by a surjection satisfying the conditions of Theorem 3, though there may be multiple such representations when two or more of the part sizes are equal.

Theorem 3. For each fixed $k, n ; n \geqslant \frac{(k+4)(k-1)}{2}+1$, there exists a ucycle of all onto functions $f:[n] \rightarrow[k]$ such that the preimage cardinality function $\left|f^{-1}\right|:\{\{1\},\{2\}, \ldots\{k\}\} \rightarrow$ $[n]$ is non-decreasing.

Proof. Similar to that of Theorem 1; the digraph is a little different, and to show connectivity we first go from an edge to another with strictly increasing PSV - and then proceed to the same target vertex as in Theorem 1.

Corollary 4. For any fixed $k \geqslant 3$, the upacking and ucovering given in Theorems 1 and 2 respectively are both asymptotically of size $\left\{\begin{array}{l}n \\ k\end{array}\right\}$, the number of partitions of $[n]$ into $k$ parts. 
Proof. This result is a special case of asymptotic results found in [5], where a threshold of $k=n^{1 / 5}$ is seen to hold for the property "partitions of size $k$ with distinct parts form a "high" fraction of all partitions of $[n]$ into $k$ parts."

\section{Universal Cycles of Partitions $\mathcal{P}(n, k)$ of $[n]$ into $k$ parts}

As in the previous section, we encode a $k$-partition of $[n]$ as a string of length $n$ containing $k$ symbols where $i$ and $j$ are in the same subset of the partition if and only if the $i$ th character in the string is the same symbol as the $j$ th character. Since the cases for $k=1$ and $k=n$ are trivial, we always assume that $2 \leqslant k<n$. For convenience, we use $\{1,2, \ldots, k\}$ as our alphabet. We refer to an encoding of a partition as a representation of that partition. Note that each $k$-partition of $[n]$ has $k$ ! different representations, and we will refer to the representation in which symbols from $[k]$ first appear, from left to right, in their natural order as being a restricted growth function or RGF representation, where the value of the RGF at any index is at most one more than its maximum over all previous indices.

Following methods outlined in [2], we construct a transition digraph $G_{n, k}$ for $\mathcal{P}(n, k)$ as follows. Let the set of vertices of $G_{n, k}$ be the set of all $k$ and $(k-1)$-partitions of $[n-1]$. There is an edge between two vertices $v$ and $w$ of $G_{n, k}$ if and only if $w$ can immediately follow $v$ in a ustring of $k$-partitions of $[n]$. That is, there is an edge from $v$ to $w$ if and only if the last $n-2$ symbols of a representation for $v$ match the first $n-2$ symbols of a representation for $w$ and the string formed by overlaying these two representations at their shared $n-2$ length substring is a representation of a $k$-partition of $[n]$. Observe that each vertex that is a $k$-partition of $[n-1]$ will have indegree $=$ outdegree $=k$, and each vertex that is a $(k-1)$-partition of $[n-1]$ will have indegree $=$ outdegree $=1$. As an example, $G_{5,3}$ is shown in Figure 1, with all vertices labeled with the RGF representation.

Now, the edges of $G_{n, k}$ are precisely the $k$-partitions of $[n]$. Furthermore, a partition $p_{1}$ can follow another partition $p_{2}$ in a ustring for $\mathcal{P}(n, k)$ if and only if the vertex at the tail of $p_{1}$ is also at the head of $p_{2}$. Thus, there is a bijection between the Eulerian cycles of $G_{n, k}$ and the ustrings of $\mathcal{P}(n, k)$.

Theorem 5. Let $n, k \in \mathbf{Z}^{+}$with $2 \leqslant k<n$, and let $G_{n, k}$ be the transition digraph for $\mathcal{P}(n, k)$. Then $G_{n, k}$ has an Eulerian cycle.

Proof. $G_{n, k}$ is balanced as remarked above, so we must show that $G_{n, k}$ is weakly connected. To do so, we show that there exists a path from any vertex of $G_{n, k}$ to the vertex $w$ with representation $(1,2, \ldots, k-1, k, k, \ldots, k)$. Accordingly, let $u$ be a vertex of $G_{n, k}$. We describe an algorithm for obtaining a path from $u$ to $w$. We first find a path from $u$ to a vertex $v$ which ends in $k$ distinct symbols. We may arrive at such a vertex in $k-1$ steps by a path $u=v_{1}, v_{2}, \ldots v_{k}$ where, for $i=1,2, \ldots, k-1$, we choose $v_{i+1}$ to be a vertex connected to $v_{i}$ such that the representations of $v_{i+1}$ end in $i+1$ distinct symbols. Note that choosing $v_{i+1}$ this way is always possible $-v_{i}$ will have representations ending in $i$ distinct symbols and if outdegree $\left(v_{i}\right)=1$ then the only possible choice for $v_{i+1}$ has representations formed by adding the missing symbol of each representation of $v_{i}$ to its 


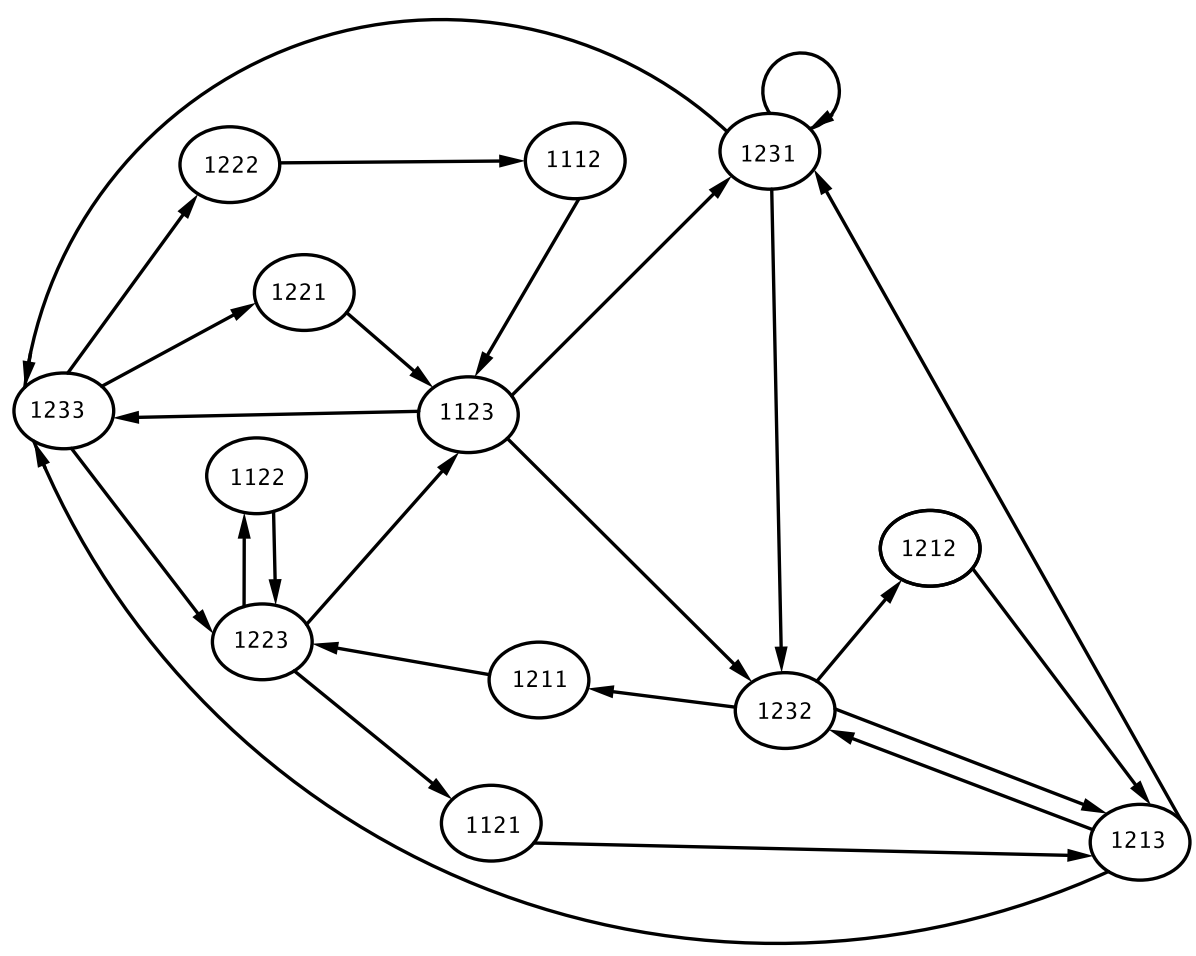

Figure 1: $G_{5,3}$

last $n-2$ symbols (the case where outdegree $\left(v_{i}\right)=k$ is clear). Now, $v_{k}$ has representations ending in $k$ distinct symbols, so for any path of length $(n-1)-k$ starting at $v_{k}$, each vertex on the path will have outdegree $=k$. Thus, there exists a path $v_{k}, v_{k+1}, \ldots, v_{n-1}$, where $v_{k+j}$ has representations whose last $j+1$ symbols are all the same $(j=0,1, \ldots,(n-1)-k)$. Then, by construction, $v_{n-1}=w$. Hence, $G_{n, k}$ is weakly connected and so it follows that $G_{n, k}$ contains an Eulerian cycle.

Hence, we know that Eulerian cycles exist in $G_{n, k}$, and therefore ustrings of $\mathcal{P}(n, k)$ exist as well. However, there may be ustrings which cannot be turned into ucycles, which occurs when the representations of the first and last partitions do not overlap correctly, i.e., they have their symbols permuted. This idea is illustrated in the Eulerian cycle in Figure 2:

If we start with the RGF representation 123 of the first vertex, then this Eulerian cycle represents the ustring 123312321, which cannot be turned into a ucycle. This example shows another important concept - once we choose the first representation to use, all other representations used are uniquely determined by the given Eulerian cycle. These observations motivate the following definitions.

Definition 6. Suppose $v$ is a vertex in $G_{n, k}$ and $r$ is a representation for $v$. Form a new string $r_{0}$ from $r$ by deleting all but the first occurence of each symbol from $r$ and appending the missing symbol to the end if $v$ is a $(k-1)$-partition. Then $r_{0}$ is a permutation of $[k]$; 


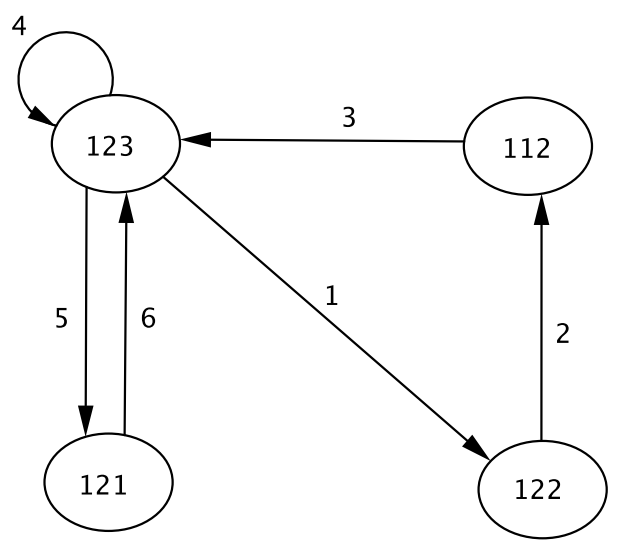

Figure 2: Eulerian cycle in $G_{4,3}$

we call $r_{0}$ the relative order of $r$.

Definition 7. Consider an edge vw for some vertices $v$ and $w$ of $G_{n, k}$. Fix a representation $r_{v}$ of $v$ and suppose it has relative order $\pi_{v}$. Suppose the corresponding representation of $w$ is $r_{w}$ with relative order $\pi_{w}$. Then $\pi_{w} \pi_{v}^{-1}$ is called the associated permutation of the edge vw.

Remark 8. We have defined the associated permutation as the $\pi \in S_{k}$ such that $\pi \pi_{v}=$ $\pi_{w}$, so that this definition is independent of the choice of representation of $v$.

The graph $G_{5,3}$ is shown again in Figure 3 with edges labeled with their associated permutations (expressed in cycle notation with fixed points supressed).

Definition 9. Let $E=e_{1}, e_{2}, \ldots, e_{S(n, k)}$ be an Eulerian cycle in $G_{n, k}$ and let $\pi_{i}$ be the associated permutation of $e_{i}, i=1,2, \ldots, S(n, k)$. We call the product $\pi_{S(n, k)} \pi_{S(n, k)-1} \cdots \pi_{2} \pi_{1}$ the permutation product of $E$.

From this definition, we get the following characterization.

Theorem 10. An Eulerian cycle $E=e_{1}, e_{2}, \ldots, e_{S(n, k)}$ in $G_{n, k}$ can be lifted to a ucycle of $\mathcal{P}(n, k)$ if and only if its permutation product is the identity.

Proof. Fix a representation $r$ of the vertex at the tail of $e_{1}$ and suppose $r$ has relative order $\tau$. $E$ can be lifted to a ucycle if and only if we arrive back at $r$ at the end of the cycle, and going through $E$ is equivalent to applying the permutation product to $\tau$.

Now, we show that the associated permutation of an edge is completely determined by the vertex at its "tail", and that only certain permutations can be associated permutations. 


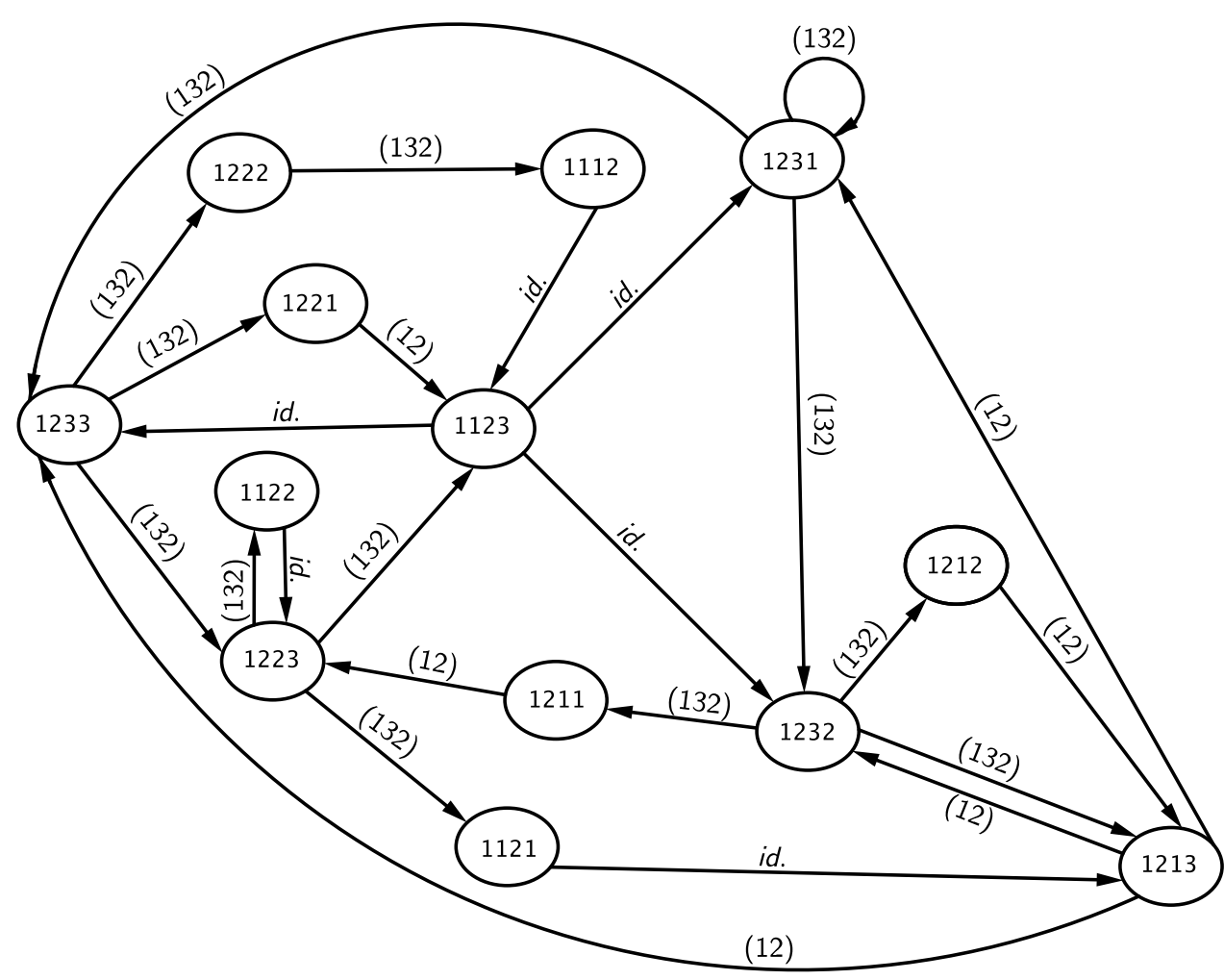

Figure 3: $G_{5,3}$ with associated permutations

Lemma 11. Let $v w_{1}$ be an edge in $G_{n, k}$, suppose $v w_{1}$ has associated permutation $\pi$. Then $\pi$ has the form $(1 j j-1 \cdots 2)$ for some $1 \leqslant j \leqslant k$, and if $v w_{2}$ is another edge from $v$, then $v w_{2}$ has associated permutation $\pi$ as well.

Proof. Let $r=v_{1} v_{2} \cdots v_{n-1}$ be the representation of $v$ with relative order $12 \cdots k$. Then there are representations $r_{1}=v_{2} \cdots v_{n-1} u_{1}$ and $r_{2}=v_{2} \cdots v_{n-1} u_{2}$ of $w_{1}$ and $w_{2}$ corresponding to $r$ under $v w_{1}$ and $v w_{2}$, respectively. Suppose that $j-1$ distinct symbols appear in $r$ after $v_{1}(=1)$ and before a second appearance of 1 (1 may only occur once in $r$ ). Since the first $n-2$ characters of both $r_{1}$ and $r_{2}$ are the same as the last $n-2$ characters of $r$, it follows that $r_{1}$ and $r_{2}$ both have relative order $23 \cdots j 1 j+1 j+2 \cdots k$. Hence, the associated permuations of $v w_{1}$ and $v w_{2}$ are both $(1 j j-1 \cdots 2)$.

Theorem 12. For $n \geqslant 3$, every Eulerian cycle of $G_{n, 2}$ can be lifted to a ucycle.

Proof. Observe that the vertex with representation $11 \cdots 1$ is the only vertex of outdegree 1 and that the edge coming out of this vertex has $i d$. as its associated permutation. All other vertices have outdegree 2 , and the two edges originating from any particular vertex both have the same associated permutation by Lemma 11. In particular, there is an even number of (12) permutations. Since $S_{2}$ is abelian, the permutation product of an Eulerian cycle will be the identity so that the result follows by Theorem 10 . 
Corollary 13. For $n \geqslant 3$, ucycles of $\mathcal{P}(n, 2)$ exist.

Proof. This follows directly from Theorem 5 and Theorem 12 .

We can also use the permutation product to determine cases when ucycles do not exist. The easiest way for this to occur is if the multiset consisting of all associated permutations in $G_{n, k}$ contains an odd number of odd permutations since this ensures that there is no ordering of the associated permutations which multiplies to the identity.

Definition 14. We call the multiset consisting of all associated permutations in $G_{n, k}$ the permutation multiset of $G_{n, k}$.

Definition 15. Let $\mathcal{O}$ be the multiset which contains all odd permutations of the permutation multiset of $G_{n, k}$. Define the parity function by

$$
\operatorname{Par}(n, k)= \begin{cases}0 & \text { if }|\mathcal{O}| \equiv 0 \bmod 2 \\ 1 & \text { if }|\mathcal{O}| \equiv 1 \bmod 2\end{cases}
$$

Lemma 16. If $\operatorname{Par}(n, k)=1$, then there does not exist a ucycle of $\mathcal{P}(n, k)$.

The following formula gives a recursive formula for calculating $\operatorname{Par}(n, k)$.

Lemma 17. The function Par $(n, k)$ satisfies the following recurrence relation:

$$
\operatorname{Par}(n, k) \equiv k \cdot \operatorname{Par}(n-1, k)+\operatorname{Par}(n-1, k-1)+\left\{\begin{array}{l}
n-2 \\
k-2
\end{array}\right\} \bmod 2
$$

with initial conditions $\operatorname{Par}(n, 2)=0$ for all $n$, and

$$
\operatorname{Par}(n, n-1)= \begin{cases}1 & \text { if } n \equiv 0 \bmod 4 \\ 0 & \text { otherwise }\end{cases}
$$

Proof. We establish a relationship between the permutation multiset of $G_{n, k}$ and those of $G_{n-1, k}$ and $G_{n-1, k-1}$. Suppose $v$ is a vertex in $G_{n, k}$, so $v$ represents a $k$ or $k-1$-partition $p$ of $[n-1]$. We consider the edge $e_{p}$ which represents $p$ in either $G_{n-1, k}$ or $G_{n-1, k-1}$. We know that the associated permutation of $e_{p}$ is determined by the location of the second occurence of the first symbol in a representation $r_{p}$ of the vertex $w_{p}$ at the tail of $e_{p}$ by Lemma 11. First, suppose $v$ represents a $k$-partition of $[n-1]$. If the first symbol does actually occur for a second time in $r_{p}$, then since there is a representation of $v$ whose first $n-2$ characters are precisely $r_{p}$, it follows that $e_{p}$ has the same associated permutation as all the edges coming from $v$. If the first symbol of $r_{p}$ does not occur a second time, then $e_{p}$ has associated permutation $(1 k k-1 \cdots 2)$. If $w_{p}$ has outdegree 1 , then the representations of $v$ do not have a second occurrence of their first symbols, and so all edges from $v$ have associated permutation $(1 k k-1 \cdots 2)$. If $w_{p}$ has outdegree $k$, then the representations of $v$ have an occurence of all symbols before a second occurence of the first symbol, so we get that the edges form $v$ have associated permutation $(1 k k-1 \cdots 2)$ again. Since each 
vertex of $G_{n, k}$ which represents a $k$-partition of $[n-1]$ has outdegree $k$, we get the term $k \cdot \operatorname{Par}(n-1, k)$.

Now, suppose $v$ represents a $k-1$-partition of $[n-1]$.

Case 1, the first symbol of $v$ appears a second time. Then either the first symbol of $r_{p}$ appears a second time, or the first symbol of $r_{p}$ is appended by following $e_{p}$ (if the second appearance in $v$ is at the last character). If the first symbol of $r_{p}$ appears a second time, then by previous reasoning $e_{p}$ has the same associated permutation as all edges from $v$. If the first symbol of $r_{p}$ does not occur a second time, then $e_{p}$ and the edges from $v$ all have associated permutation $(1 k k-1 \cdots 2)$.

Case 2, the first symbol of $v$ does not appear a second time. Then the first symbol of $r_{p}$ does not appear a second time, and so $e_{p}$ must have associated permutation $(1 k-1 k-$ $2 \cdots 2)$. However, in this case $v$ has associated permutation $(1 k k-1 \cdots 2)$. Note that since the first symbol of $v$ does not appear a second time, the last $n-2$ characters of $v$ represent a $k-2$-partition of $[n-2]$, so this case occurs exactly $\left\{\begin{array}{c}n-2 \\ k-2\end{array}\right\}$ times. Thus, we have $\left\{\begin{array}{c}n-2 \\ k-2\end{array}\right\}$ partitions that either switch from even to odd or odd to even; in either case adding $\left\{\begin{array}{l}n-2 \\ k-2\end{array}\right\}$ affects the parity in the desired manner.

Thus, each vertex in $G_{n, k}$ which represents a $k-1$-partition of [ $\left.n-1\right]$ has the same associated permutation as it does in the graph $G_{n-1, k-1}$ except for $\left\{\begin{array}{l}n-2 \\ k-2\end{array}\right\}$ permutations which change sign. Since each such vertex has outdegree 1, we get the $\operatorname{Par}(n-1, k-1)+\left\{\begin{array}{l}n-2 \\ k-2\end{array}\right\}$ term.

Finally, the initial condition $\operatorname{Par}(n, 2)=0$ for all $n$ follows from Theorem 12 , and the initial condition

$$
\operatorname{Par}(n, n-1)=\left\{\begin{array}{lc}
1 & \text { if } n \equiv 0 \bmod 4 \\
0 & \text { otherwise }
\end{array}\right.
$$

follows from the remark following Theorem 5 in [4]

Corollary 18. For $n \geqslant 4$ and $2 \leqslant k<n$,

$$
\operatorname{Par}(n, k) \equiv \begin{cases}0 \bmod 2 & \text { if } n \text { is odd } \\
\left\{\begin{array}{l}
n-2 \\
k-2
\end{array}\right\} \bmod 2 & \text { if } n \text { is even }\end{cases}
$$

Proof. We proceed by induction. For the base cases, we show that the initial conditions of the recursion in Corollary 17 satisfy Equation (2). If we define $\left\{\begin{array}{l}n \\ 0\end{array}\right\}:=0$ for all $n$, then Equation ( 2) yields $\operatorname{Par}(n, 2)=0$ for all $n$. Also, if $n$ is even, then Equation 2 yields

$$
\begin{aligned}
\operatorname{Par}(n, n-1)=\left\{\begin{array}{l}
n-2 \\
n-3
\end{array}\right\}=\left(\begin{array}{c}
n-2 \\
2
\end{array}\right) & =\frac{(n-2)(n-3)}{2} \\
& \equiv \begin{cases}0 \bmod 2 \text { if } n \equiv 2 \bmod 4 \\
1 \bmod 2 \text { if } n \equiv 0 \bmod 4\end{cases}
\end{aligned}
$$

as desired. 
Now, suppose that (2) holds for $\operatorname{Par}(n-1, k)$ and $\operatorname{Par}(n-1, k-1)$. If $n$ is odd then $n-1$ is even, so by (1), the induction hypothesis, and the fundamental Stirling number recurrence,

$$
\begin{aligned}
& \operatorname{Par}(n, k) \equiv k \cdot \operatorname{Par}(n-1, k)+\operatorname{Par}(n-1, k-1)+\left\{\begin{array}{l}
n-2 \\
k-2
\end{array}\right\} \quad \bmod 2 \\
& \equiv k \cdot\left\{\begin{array}{l}
n-3 \\
k-2
\end{array}\right\}+\left\{\begin{array}{l}
n-3 \\
k-3
\end{array}\right\}+\left\{\begin{array}{l}
n-2 \\
k-2
\end{array}\right\} \quad \bmod 2 \\
& \equiv 2\left\{\begin{array}{l}
n-3 \\
k-2
\end{array}\right\}+\left\{\begin{array}{l}
n-2 \\
k-2
\end{array}\right\}+\left\{\begin{array}{l}
n-2 \\
k-2
\end{array}\right\} \quad \bmod 2 \\
& \equiv 2\left\{\begin{array}{l}
n-2 \\
k-2
\end{array}\right\} \quad \bmod 2 \\
& \equiv 0 \quad \bmod 2
\end{aligned}
$$

If $n$ is even, then $n-1$ is odd and so $\operatorname{Par}(n-1, k)=\operatorname{Par}(n-1, k-1)=0$. Hence, $\operatorname{Par}(n, k) \equiv\left\{\begin{array}{c}n-2 \\ k-2\end{array}\right\} \bmod 2$ by (1).

Corollary 19. If $n$ is even and $\left\{\begin{array}{l}n-2 \\ k-2\end{array}\right\}$ is odd, there does not exist a ucycle for $k$-partitions of $[n]$.

Proof. This follows directly from Corollary 18.

Corollary 20. For each $k \geqslant 3, k \not \equiv 1(\bmod 4)$, there are infinitely many even $n$ 's for which ucycles of $\mathcal{P}(n, k)$ do not exist.

Proof. To prove the corollary, we use the well-known fact [1] that

$$
\left\{\begin{array}{l}
n \\
k
\end{array}\right\}=\left(\begin{array}{c}
n-\lfloor k / 2\rfloor-1 \\
n-k
\end{array}\right) \quad(\bmod 2)
$$

Thus

$$
\left\{\begin{array}{l}
n-2 \\
k-2
\end{array}\right\}=\left(\begin{array}{c}
n-\lfloor k / 2\rfloor-2 \\
n-k
\end{array}\right)=\left(\begin{array}{l}
n-\lfloor k / 2\rfloor-2 \\
k-\lfloor k / 2\rfloor-2
\end{array}\right) \quad(\bmod 2) .
$$

We also know (see, e.g., Theorem 4.1 .10 at http://www.cs.columbia.edu/ cs4205/ files/CM4.pdf) that

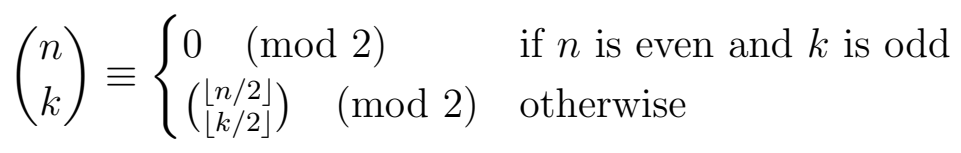

If $k=4 s+1$, then

$$
\left\{\begin{array}{l}
n-2 \\
k-2
\end{array}\right\} \equiv\left(\begin{array}{c}
n-2 s-2 \\
2 s-1
\end{array}\right) \quad(\bmod 2)
$$

and no conclusion may be reached due to (4), since this is an ( $\left.\begin{array}{c}\text { even } \\ \text { odd }\end{array}\right)$ situation. Using the above facts, it is easy to check that for $k=3,4,6$, ucycles do not exist for any even $k$. For 
$k=7,\left\{\begin{array}{c}n-2 \\ 5\end{array}\right\} \equiv\left(\begin{array}{c}n-5 \\ 2\end{array}\right)=(n-5)(n-6) / 2(\bmod 2)$ and thus ucycles do not exist if $n \equiv 0$ $(\bmod 4)$. The same is true for $k=8$. For $k=10$, by $(3)$ and $(4)$, we see that ucycles do not exist when $\lfloor(n-7) / 2\rfloor$ is odd, or when $n \equiv 2(\bmod 4)$. We treat the general cases $k=4 s, k=4 s+2, k=4 s+3$ next, simultaneously. In these three cases respectively we have, by (3),

$$
\begin{gathered}
\left\{\begin{array}{l}
n-2 \\
k-2
\end{array}\right\} \equiv\left(\begin{array}{c}
n-2 s-2 \\
2 s-2
\end{array}\right) \text { or } \\
\left\{\begin{array}{l}
n-2 \\
k-2
\end{array}\right\} \equiv\left(\begin{array}{c}
n-2 s-3 \\
2 s-1
\end{array}\right) \text { or } \\
\left\{\begin{array}{l}
n-2 \\
k-2
\end{array}\right\} \equiv\left(\begin{array}{c}
n-2 s-3 \\
2 s
\end{array}\right) .
\end{gathered}
$$

In each of these cases we cannot conclude summarily that the Stirling number $\left\{\begin{array}{l}n-2 \\ k-2\end{array}\right\}$ is even - since we never have an ( $\left.\begin{array}{c}\text { even } \\ \text { odd }\end{array}\right)$ situation. Let us proceed to iterate (4) until, after roughly $\log \left(\frac{k}{2}\right)$ steps, we arrive at an $\left(\begin{array}{c}M \\ 1\end{array}\right)=M$ term for some $m \in \mathbb{Z}^{+}$. We claim that for each odd $M$, it is possible to choose at least one starting $n$ for which the above iterations never contain an ( $\left.\begin{array}{c}\text { even } \\ \text { odd }\end{array}\right)$ term, so that we can deduce that a ucycle doesn't exist. Starting at $\left(\begin{array}{c}M \\ 1\end{array}\right)$ we manipulate the backward path of equivalences to be

$$
\left(\begin{array}{c}
2 M+1 \\
a_{1}
\end{array}\right),\left(\begin{array}{c}
4 M+3 \\
a_{2}
\end{array}\right),\left(\begin{array}{c}
8 M+7 \\
a_{3}
\end{array}\right)
$$

etc., none of which are of the form $\left(\begin{array}{c}\text { even } \\ \text { odd }\end{array}\right)$, where the $a_{i}$ are determined by the value of $k+\lfloor k / 2\rfloor-2$ in the starting binomial coefficient. Now if $r$ iterations of (4) are needed to get to $M$, then the initial binomial coefficient must equal

$$
\left(\begin{array}{l}
n-\lfloor k / 2\rfloor-2 \\
k-\lfloor k / 2\rfloor-2
\end{array}\right)=\left(\begin{array}{c}
2^{r}+2^{r}-1 \\
k-\left\lfloor\frac{k}{2}\right\rfloor-2
\end{array}\right)
$$

so that the required solution is $n \equiv\lfloor k / 2\rfloor+1\left(\bmod 2^{r+1}\right)$. This completes the proof, though it should be noted that we have not entirely characterized those $n$ for which ucycles do not exist in terms of $k$, since if $a_{i}$ is even there are two choices for the "top" binomial coefficient. Overall, however, the solutions get progressively sparser due to the fact [8] that "most binomial coefficients are even".

In practice, for fixed $k$, the task of identifying suitable $n$ 's can be done exactly, as seen by the following example.

Example 21. Ucycles of $\mathcal{P}(n, 23)$ do not exist for $n \equiv 8,12(\bmod 16)$.

Proof. We have

$$
\left\{\begin{array}{c}
n-2 \\
23-2
\end{array}\right\} \equiv\left(\begin{array}{c}
n-13 \\
10
\end{array}\right) \equiv\left(\begin{array}{c}
\left\lfloor\frac{n-13}{2}\right\rfloor \\
5
\end{array}\right) \equiv\left(\begin{array}{l}
a \\
2
\end{array}\right) \equiv\left(\begin{array}{l}
b \\
1
\end{array}\right)
$$


where $a, b$ are respectively the "twice- and thrice-iterated half-floors" of $n-13$. For any odd $b \geqslant 1$, moreover, we must have $a=2 b$ or $a=2 b+1$. Thus

$$
\left\lfloor\frac{n-13}{2}\right\rfloor=4 b, 4 b+1,4 b+2, \text { or } 4 b+3,
$$

whence we get $n-13=8 b, \ldots, 8 b+7$, or $n=13+8 b, \ldots, 20+8 b$. Eliminating the odd $n$ 's leads to $n=14+8 b, 16+8 b, 18+8 b, 20+8 b$, or

$$
n=\frac{16(b+1)}{2}+6, \frac{16(b+1)}{2}+8, \frac{16(b+1)}{2}+10, \frac{16(b+1)}{2}+12 .
$$

The solutions that are congruent to 6 or 10 ( $\bmod 16)$ can be checked to teminate prematurely in an $\left(\begin{array}{c}\text { even } \\ \text { odd }\end{array}\right)$ coefficient. This proves the claim.

\section{Open Questions}

We can ask the following:

(a) The smallest case remaining after our investigation is the one for $n=5, k=3$, for which we have found the ucycle 3112311123213233112131322. All other cases for $n=5$ and $n=6$ are solved. This leads to the question: What is the best result that can be proved along the following lines "For $n \geqslant 3$ and $3 \leqslant k<n$, ucycles of $k$-partitions of $[n]$ exist if and only if $n$ is odd?"

(b) In general, how can one use Theorem 10 to prove results on existence of ucycles (rather than non-existence)?

(c) Even if ucycles of $\mathcal{P}(n, k)$ may not exist, when it it true that ucycles exist for $\mathcal{P}(n, s, t)$, the set of partitions of $[n]$ into between $s$ and $t$ parts; $s<t$ ?

(d) Throughout this paper, we have insisted on having the alphabet size equal $k$. How do our results change if we relax this condition?

\section{Acknowledgments}

The research of all the authors was supported by NSF Grant 1263009. We thank the anonymous referee for their careful remarks, and a vital suggestion that vastly improved Corollary 20.

\section{References}

[1] O-Y. Chan and D. Manna. Congruences for Stirling numbers of the second kind. Contemporary Math., 517, 97-11, 2010.

[2] F. Chung, P. Diaconis, and R. Graham. Universal cycles for combinatorial structures. Discrete Math., 110, 43-59, 1992.

[3] D. Curtis, T. Hines, G. Hurlbert, and T. Moyer. Near-universal cycles for subsets exist. SIAM J. Discrete Math., 23, 1441-1449, 2009. 
[4] K. Casteels and B. Stevens. Universal cycles of ( $n-1)$-partitions of an $n$-set. Discrete Math., 309, 5332-5340, 2009.

[5] É. Czabarka, M. Marsili, and L. A. Székely. Threshold functions for distinct parts: revisiting Erdős-Lehner. In Information Theory, Combinatorics, and Search Theory (in Memory of Rudolph Ahlswede), eds. H. Aydinian, F. Cicalese, C. Deppe, volume 7777 of Lecture Notes in Computer Science, pages 463-471. Springer-Verlag, 2013.

[6] M. Dewar and B. Stevens. Gray Codes, Universal Cycles, and Configuration Orderings. Springer Verlag, New York, 2012.

[7] A. Elks, A. Godbole, and S. McInturff. Universal Cycles for 2- and 3-Partitions of [n]. To appear in Cong. Numer., 2015+.

[8] H. Harborth. Number of odd binomial coefficients. Proc. Amer. Math. Soc., 62, 19-22, 1977.

[9] G. Hurlbert. On universal cycles for $k$-subsets of an $n$-set. SIAM J. Discrete Math., 7, 598-604, 1994.

[10] B. LaBounty-Lay, A. Bechel, and A. Godbole. Universal cycles of discrete functions. Cong. Numer., 189, 121-128, 2008. 